\title{
A Novel Test of Implicit Memory; an Eye Tracking Study
}

\author{
Jonathan Chung* ${ }^{1}$, Sarah Chau ${ }^{1}$, Krista Lanctôt ${ }^{1}$, Nathan Herrmann ${ }^{1}$, Moshe Eizenman ${ }^{1}$
}

Accepted $15^{\text {th }}$ August 2014

\begin{abstract}
Novelty preference in visual scanning behaviour is used to test implicit memory in patients with Alzheimer's disease (AD). During the test, subjects are presented with slides that include both novel images and images that were seen before (repeated images). Slides are presented sequentially and the number of slides between the first and second presentations of repeated images is varied to mask the purpose of the test. The normalised average glance duration (N-AGD) on repeated images (the bias towards novelty) was used to measure novelty preference. Data from 10 young controls showed that the bias towards novelty is reduced as the number of slides between the first and second presentations of repeated images is increased. A group of 17 patients with AD showed no significant bias towards novelty while a group of 21 age matched controls do exhibit such bias $(\mathrm{t}(20)=6.16, \mathrm{p}<0.001)$. The data suggest that patients with $\mathrm{AD}$ have no preference to novel images and support the idea that $\mathrm{AD}$ affects implicit memory. The receiver operator characteristics of the bias towards novelty showed that patients with $\mathrm{AD}$ and age-matched controls can be differentiated with both high sensitivity and high specificity.
\end{abstract}

Keywords: Eyes, Alzheimer's Disease, Psychology, Cognition, Neuroscience

\section{Introduction}

Implicit memory is a form of a subconscious remembering that can be solidified through priming [1], [2]. Typically, implicit memory is assessed by tests such as the lexical decisions task [3] and the word-stem completion task [4] that require subject cooperation. The visual paired comparison task [5] is another test of implicit memory in which two visual stimuli, one that is novel and one that was previously seen, are presented simultaneously and the length of time that subjects look at each stimulus is measured. Infants [5] and adults [6] typically look longer at novel images than at images that were previously seen (repeated images). This phenomenon is called the novelty preference and it is assumed to be associated with implicit memory [7]. Since novelty preference can be tested with minimal subject cooperation, it can be used to test implicit memory in patients with Alzheimer Disease (AD) who have difficulties to follow instructions and can be frustrated by standard cognitive tests. Previous tests of implicit memory in patients with $\mathrm{AD}$ have shown mixed results. A review by Fleischman and Gabrieli [8] suggested that patients with $\mathrm{AD}$ have comparable implicit memory capabilities to age-matched controls. However, this suggestion was criticised when a music preference-based test showed that patients with AD have no implicit memory [9]. In this paper we describe a novel method to test for novelty preference and use the method to evaluate implicit memory in patients with $\mathrm{AD}$.

During the test, subjects look at novel and repeated images and differences in their visual scanning behaviour (VSB) on these two types of images are used to measure novelty preference. To

\# This paper has been presented at the International Conference on Advanced Technology\&Sciences (ICAT'14) held in Antalya (Turkey), August 12-15, 2014.

${ }^{1}$ University of Toronto, Canada

*Corresponding Author: Email: jono.chung@mail.utoronto.ca minimise the chance that subjects can recognise the presentation pattern and consciously change their VSB, we varied the number of slides between the first and second presentations of the repeated images throughout the test. Some parameters of the new method (e.g. the VSB parameter that best describe novelty preference) were determined from experiments with young controls. The new method was then used to estimate novelty preference in patients with $\mathrm{AD}$ and age-matched controls.

\section{Methods}

Visual Attention Scanning Technology (VAST, EL-MAR Inc. Toronto, Ontario, Canada) was used to obtain subjects' eye gaze positions. VAST incorporates a binocular gaze estimation system [10] to estimate and record gaze position, a display to present visual stimuli and a monitoring station to control and supervise the test. Estimates of gaze positions are segmented into fixations that are linked to images that are presented on the display. During the test, subjects looked at either 60 slides (young controls) or 48 slides (patients with $\mathrm{AD}$ and age-matched controls). Each slide was presented for 10.5 seconds with a 1 second interval between slides. Each slide had four images of neutral objects that were arranged in a $2 \times 2$ configuration. Images of neutral objects were selected from the International Affective Picturing system (IAPS) database with valences from 4.0 to 6.0 and arousals of less than 4.5 [11]. Images on the same slide had similar sizes and complexities.

During the tests, two types of slides were presented: slides with four novel images and slides with two novel and two repeated images. In this paper, the repeated images are often referred to as $n$-back where " $n$ " is the number of slides between the first and second presentations of the images. Images that were repeated appeared in the same locations on the slide during the first and second presentations. For young controls the number of slides between the first and second presentations varied between 1, 5, 10 or 15 slides (1-back, 5-back, 10-back and 15-back). For 
patients with $\mathrm{AD}$ and age-matched controls the number of slides between the first and second presentations was either 1 or 2 . For young controls, each of the test conditions (e.g., 1-back, 5-back) was repeated 12 times and for patients with $\mathrm{AD}$ and age-matched controls each of the test conditions was repeated 16 times.

Based on the study by Snyder et. al. [7] who found that the temporal components of processing are important in the investigation of novelty preference, we evaluated biases in VSB parameters during both early and late visual processing. For early visual processing (EVP), we calculated the duration of all the fixations during the first visit to an image. For late visual processing (LVP), we calculated the duration of all the fixations during later visits (i.e., all the visits that followed the first visit). In a previous study we have successfully used fixation time, fixation frequency and average glance duration to study biases in VSB of patients with depression [12]. Fixation time is defined as the duration of all fixations on an image. Fixation frequency is defined as the number of times that each image was visited and the average glance duration is defined as the average duration of a visit to an image. We will evaluate the suitability of these parameters as well as EVP and LVP to serve as physiological markers for novelty preference.

Many high-level cognitive processes (mood, goals, etc.) and lowlevel visual processes (image properties such as colours, contrast, etc.,), which are not associated with novelty preference, affect the subjects' VSB. To reduce the effects of these processes on the estimation of novelty preference all VSB parameters on slides that include both novel and repeated images were normalised (Equation 1).

Normalised $v p=0.25-\frac{\frac{1}{2}\left(v p_{\text {repeat }-a}+v p_{\text {repeat }-b}\right)}{\sum_{i=1}^{4} v p_{i}}$

Where $v p=V S B$ parameter

$i=$ image on a slide

$v p_{\text {repeat-a }}$ and $v p_{\text {repeat- } b}$ are the repeated images on the slide If a VSB parameter on repeated images is similar to that on novel images, the normalised VSB parameter will be zero (no novelty preference). If memory of previously seen images reduces the attention allocated to repeated images (indication of a novelty preference) the normalised VSB parameters will be positive. The larger the magnitude of the normalised VSB parameters the larger is the difference between the attention allocated to novel images and the attention allocated to repeated images. For each subject, the means and the standard deviations of the normalised VSB parameters were calculated.

During the study, data were collected from 10 university students (age $25.00 \pm 5.30$ ) who served as young controls, 21 age-matched controls (Mini Mental State Examination score $>=26$, Age 71.65 \pm 9.26 ) and 17 patients with $\mathrm{AD}$ (Age $79.05 \pm 8.92$ ).

The data was explored with descriptive analyses that included the means and standard deviations of the normalised VSB parameters. Differences in VSB between groups and within groups were explored with repeated measures ANOVAs. For the study of the young control group the ANOVA analysis was a $1 \mathrm{x} 4$ design with 1 diagnostic group (young controls) and 4 test conditions (1-back, 5-back, 10-back and 15-back). For the study of the patients with $\mathrm{AD}$ the ANOVA analysis was a $2 \times 2$ design with 2 diagnostic groups (patients with $\mathrm{AD}$, age-matched controls) and 2 test conditions (1-back, 2-back). Where significant differences are found, post-hoc two-tailed t-tests with Bonferroni correction are performed. Significance levels for all statistical tests were set to $\mathrm{p}=0.05$.

\section{Results}

\subsection{Analysis of data from young controls}

The data from the young control group were analysed to determine: a) the VSB parameter that best indicates novelty preference in a group of subjects that should exhibit such preference [6], b) the robustness of this parameter to differences in the number of slides between the first and second presentations of repeated images, and c) the expected error in the estimation of the mean of this parameter for each subject.

The normalised means and standard deviations for the five VSB parameters that were described in the Methods section are shown in Table I. The third column in Table I provides the probability of accepting the hypothesis that the group of young controls has no novelty preference (i.e., reaching a false conclusion regarding this group of subjects). The data in Table I suggest that: a) VSB parameters that integrate information over the full length of the stimulus presentation (e.g., fixation time) have lower probabilities of reaching a false conclusion than VSB parameters that are associated with either early or late processing, and b) the probability of reaching a false conclusion is lowest when the parameter normalised average glance duration is used. In the new method, the normalised average glance duration (N-AGD) is used to measure the bias towards novelty.

Table I. Analysis of VSB parameters (One-back images)

\begin{tabular}{|c|c|c|}
\hline VSB Parameter & $\begin{array}{c}\text { Normalised VSB } \\
\text { Parameter }\end{array}$ & $\begin{array}{c}\text { Probability of no } \\
\text { novelty preference }\end{array}$ \\
\hline Fixation Time & $0.0936 \pm 0.0444$ & $9.26 \times 10^{-5}$ \\
\hline Average Glance Duration & $0.0816 \pm 0.0362$ & $5.53 \times 10^{-5}$ \\
\hline Fixation Frequency & $0.0256 \pm 0.0313$ & $3.00 \times 10^{-2}$ \\
\hline Early Processing & $0.0583 \pm 0.0443$ & $2.40 \times 10^{-3}$ \\
\hline Late Processing & $0.0671 \pm 0.0502$ & $2.20 \times 10^{-3}$ \\
\hline
\end{tabular}

The sensitivity of our measure for novelty preference (N-AGD) to the number of slides between the first and second presentations of repeated images is described in Table II. The second and third columns of Table II show that for all test conditions (1-back to 15 -back) the group of young adults demonstrated significantly ( $\alpha$ $<0.05$ ) positive biases towards novelty. However, the magnitude of the bias towards novelty decreases as the number of slides between the first and second presentations of the repeated images increases.

Table II. Bias towards novelty (R-AGD) on n-back tests

\begin{tabular}{|c|c|c|}
\hline $\begin{array}{c}\text { Test } \\
\text { Condition }\end{array}$ & Bias towards novelty & $\begin{array}{c}\text { Probability of no } \\
\text { novelty preference }\end{array}$ \\
\hline 1-back & $0.0816 \pm 0.0362$ & $5.53 \times 10^{-5}$ \\
\hline 5-back & $0.0665 \pm 0.0279$ & $3.58 \times 10^{-5}$ \\
\hline 10-back & $0.0607 \pm 0.0451$ & $2.13 \times 10^{-3}$ \\
\hline 15-back & $0.0318 \pm 0.0360$ & $2.09 \times 10^{-2}$ \\
\hline
\end{tabular}

Using a repeated measures ANOVA, the biases towards novelty in the 1, 5, 10 and 15-back test conditions were found to be significantly different $(F(3,27)=9.22, p<0.05)$. Post-hoc analysis revealed significant differences between the N-AGD on 1-back and 15-back ( $\mathrm{p}<0.01)$ and between 5-back and 15-back $(p=0.005)$. Since the magnitude of our measure of bias towards novelty decreases as the number of slides between presentations increases, we only used the 1-back and 2-back conditions in our study of patients with AD.

For each young control subject, the estimate of the mean bias towards novelty was obtained by averaging six estimates of $\mathrm{N}$ AGDs in each of the test conditions. The range of the standard errors of the mean estimates of N-AGD, for all test conditions and for all subjects, was from 0.0130 to 0.0306 . When compared with the magnitude of the mean bias towards novelty (Table II), 
the standard error of the mean is relatively large. To reduce the standard error of the mean in the study of patients with $\mathrm{AD}$, the number of estimates of $\mathrm{N}-\mathrm{AGD}$ for each test condition was increased from 6 to 16 .

\subsection{Bias towards novelty in patients with $\mathrm{AD}$}

The mean bias towards novelty for the 1-back and 2-back conditions, for patients with $\mathrm{AD}$ and age-matched controls are shown in Table III. Patients with AD did not show positive bias towards novelty in either the 1-back $(\mathrm{t}(16)=1.25, \mathrm{p}=0.227)$ or the 2-back test conditions $(\mathrm{t}(16)=1.19, \mathrm{p}=0.250)$. Age matched controls show significant positive biases towards novelty in both the 1-back $(\mathrm{t}(20)=8.07, \mathrm{p}<0.001)$ and 2-back test conditions $(\mathrm{t}(20)=6.16, \mathrm{p}<0.001)$.

Table III. Bias towards novelty on patients with $\mathrm{AD}$ and age-matched controls

\begin{tabular}{|c|c|c|}
\hline Test & Patients with AD & Age-matched controls \\
\hline 1-back & $0.00940 \pm 0.0309$ & $0.0452 \pm 0.0257$ \\
\hline 2-back & $0.00550 \pm 0.0190$ & $0.0390 \pm 0.0290$ \\
\hline
\end{tabular}

Using repeated measures ANOVA, patients with $\mathrm{AD}$ and agedmatched controls had significantly different biases towards novelty $(\mathrm{F}(1,16)=15.10, \mathrm{p}<0.05)$. Post-hoc analysis showed significant differences between the groups for 1-back $(\mathrm{t}(36)=$ $3.90, \mathrm{p}<0.001)$ and 2-back conditions $(\mathrm{t}(36)=3.90, \mathrm{p}<<$ 0.001). The above tests suggest that patients with $\mathrm{AD}$ have no novelty preference (bias towards novelty is not significantly different from zero) while age-matched controls do exhibit such preference (bias towards novelty is significantly different from zero).

Since the bias towards novelty is significantly different in patients with $\mathrm{AD}$ and age-matched controls, receiver operating characteristics between individual patients with $\mathrm{AD}$ and agematched controls was studied. Using the average of the bias towards novelty from the 1-back and 2-back test conditions the sensitivity and specificity was tested with a receiver operating curve (ROC) which was shown in (Fig. 1)

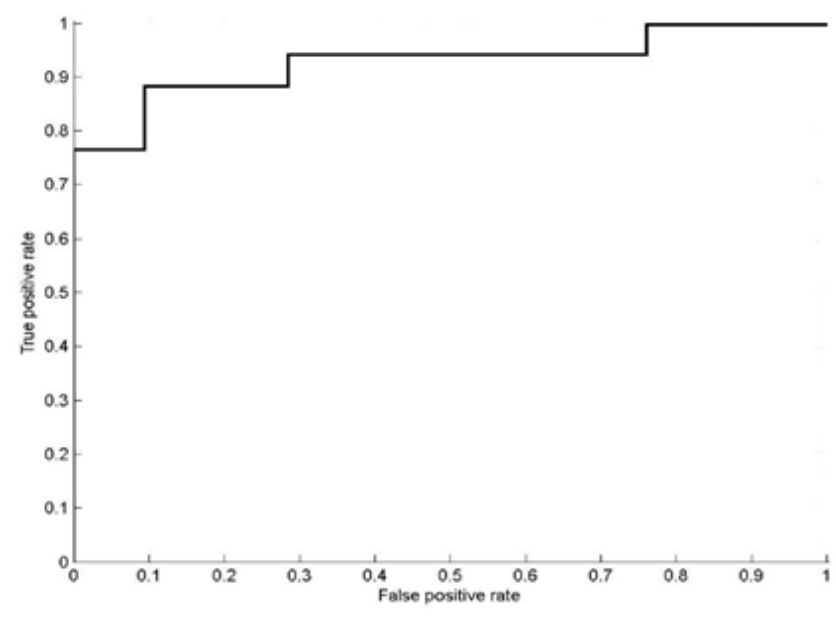

Figure 1. ROC of bias towards novelty

The ROC has both high sensitivity and high specificity (area under the curve is 0.927 ). If for example, the false positive rate is set to $5 \%$ (specificity $95 \%$, the threshold is set to 0.0143 ) the true positive rate (sensitivity) is $77 \%$. The sensitivity can be increased (specificity is decreased) by increasing the threshold. If for example, the sensitivity is increased to $88 \%$ (threshold is set to 0.0178 ) the false positive rate is $10 \%$.

\section{Discussion}

A new method to assess novelty preference in subjects was described. The method is based on the analysis of differences in visual scanning behaviour when subjects view novel images and images that have already been seen. Using the normalised average glance duration as a measure of novelty preference, the data in this paper suggest that unlike age-matched controls who demonstrate preference to novel images over repeated images, $\mathrm{AD}$ patients do not show such preference. The receiver operating characteristics of the bias towards novelty to differentiate between patients with $\mathrm{AD}$ and age-matched controls has both high sensitivity and high specificity.

The data in this study suggest that AD affects implicit memory, but it is not clear when in the course of the disease such affects take place. If $\mathrm{AD}$ affects implicit memory in the early stages of the disease the method described in this paper can be used for early detection of subjects that are at risk of developing AD. We plan to study the bias towards novelty in patients with mild cognitive impairment (MCI), which is an early form of dementia and is recognised as a risk factor of AD.If patients with MCI who do not become patients with AD show bias towards novelty, while MCI patients who become patients with AD show no such bias, the method described in this paper can be used to differentiate between these two groups.

\section{Acknowledgements}

This study was supported by the Visual Science Research Program (VSRP) at the University of Toronto, the National Research Council of Canada (NSERC), the Consortium of Canadian Centres for Clinical Cognitive Research (C5R) and the Estate of Vimy Lena Elizabeth Coleman Fund through Sunnybrook Health Sciences Centre

\section{References}

[1] H. L. Roediger, "Implicit memory. Retention without remembering," Am Psychol, vol. 45, pp. 1043-56, Sep. 1990.

[2] E. Tulving and D. L. Schacter, "Priming and human memory systems," Science, vol. 247, pp. 301-306, Jan. 1990.

[3] D. E. Meyer and R. W. Schvaneveldt, "Facilitation in recognizing pairs of words: evidence of a dependence between retrieval operations," J. Exp. Psychol., vol. 90, p. 227, 1971.

[4] E. K. Warrington and L. Weiskrantz, "Amnesic syndrome: Consolidation or retrieval?," Nature, 1970.

[5] R. L. Fantz, "Visual experience in infants: decreased attention to familiar patterns relative to novel ones," Science, vol. 146, pp. 668-70, Oct. 1964.

[6] R. D. McKee and L. R. Squire, "On the development of declarative memory," J Exp Psychol Learn Mem Cogn, vol. 19, pp. 397-404, Mar. 1993.

[7] K. A. Snyder, M. P. Blank, and C. J. Marsolek, "What form of memory underlies novelty preferences?," Psychon Bull Rev, vol. 15, pp. 315-21, Apr. 2008.

[8] D. A. Fleischman and J. D. Gabrieli, "Repetition priming in normal aging and Alzheimer's disease: a review of findings and theories," Psychol. Aging, vol. 13, p. 88, 1998.

[9] A. R. Halpern and M. G. O'Connor, "Implicit memory for music in Alzheimer's disease," Neuropsychology, vol. 14, pp. 391-7, Jul. 2000. 
[10] E. D. Guestrin and E. Eizenman, "General theory of remote gaze estimation using the pupil center and corneal reflections," Biomed. Eng. IEEE Trans. On, vol. 53, pp. 1124-1133, 2006.

[11] P. Lang, M. Bradley, and N. M. Buthbert, "International affective picture system (IAPS): Affective ratings of pictures and instruction manual," 2008.

[12] M. Eizenman, L. H. Yu, L. Grupp, E. Eizenman, M. Ellenbogen, M. Gemar, and R. D. Levitan, "A naturalistic visual scanning approach to assess selective attention in major depressive disorder," Psychiatry Res., vol. 118, pp. 117-128, 302003. 